\title{
Medida de estilos de aprendizagem para o ensino fundamental
}

\author{
Katya Luciane de Oliveira \\ Universidade Estadual de Londrina - Londrina - PR - Brasil \\ Acácia Aparecida Angeli dos Santos \\ Universidade São Francisco - Itatiba - SP - Brasil \\ Fabio Alexandre Pereira Scacchetti \\ Universidade Tecnológica Federal do Paraná - Apucarana - PR - Brasil
}

\begin{abstract}
Resumo
A presente pesquisa visou levantar evidências de validade relativa à análise da estrutura interna dos itens de uma escala de estilos de aprendizagem, bem como identificar os estilos de aprendizagem desses alunos. Participaram 565 alunos dos $3^{\circ}$ ao $9^{\circ}$ anos do ensino fundamental público do estado do Paraná. Utilizou-se uma Escala de Avaliação de Estilos de Aprendizagem, com 80 itens, aplicada coletivamente. A análise fatorial, por componentes principais e com rotação varimax, indicou estrutura de quatro Dimensões para a escala. As Dimensões se agruparam da seguinte forma: Dimensão 1 - Condições ambientais e condições pessoais, com 22 itens; Dimensão 2 - Condições sociais, com 11 itens; Dimensão 3 - Condições instrumentais, com 11 itens e Dimensão 4 - Condições de atividade; os itens desta dimensão não se agruparam do modo esperado, por isso novos estudos deverão ser realizados, visando o refinamento do instrumento. As condições ambientais e pessoais foram os estilos predominantes nesta amostra.
\end{abstract}

Palavras-chave: Aprendizagem; estilos cognitivos; avaliação psicológica.

\section{Learning styles measurement for Elementary Education}

\begin{abstract}
This research aimed to collect evidence of validity on the analysis of the internal structure of items in a range of learning styles and to identify learning styles of these students. About 565 students in the 3rd to the 9th years of elementary school public participated in the state of Paraná. We used a Learning Styles Assessment Scale with 80 items, applied collectively. Factor analysis by principal and varimax rotation components, are divided in four structure dimensions for scale. The dimensions are grouped as follows: Dimension 1 - Environmental conditions and personal, with 22 items; Dimension 2 - Social conditions, with 11 items; Dimension 3 - instrumental conditions, with 11 items and size 4 - Conditions of activity; the items in this dimension not grouped in the expected manner, so further studies should be conducted, aimed to refining the instrument. Environmental and personal conditions were the predominant styles in this sample.
\end{abstract}

Keywords: Learning; Cognitive styles; Psychological assessment.

\section{Medida de estilos de aprendizaje para la enseñanza fundamental}

\section{Resumen}

La presente investigación tuvo por objetivo levantar evidencias de validad relativa al análisis de la estructura interna de los ítems de una escala de estilos de aprendizaje, así como identificar los estilos de aprendizaje de esos alumnos. Participaron 565 alumnos de los $3^{\text {er }}$ al $9^{\circ}$ curso de la enseñanza básica pública del estado de Paraná. Se utilizó una Escala de Evaluación de Estilos de Aprendizaje, con 80 ítems, aplicada colectivamente. El análisis factorial, por componentes principales y con rotación varimax, indicó estructura de cuatro Dimensiones para la escala. Las Dimensiones se agruparon de la siguiente forma: Dimensión 1 - Condiciones ambientales y condiciones personales, con 22 ítems; Dimensión 2 - Condiciones sociales, con 11 ítems; Dimensión 3 - Condiciones instrumentales, con 11 ítems y Dimensión 4 - Condiciones de actividad; los ítems de esta dimensión no se agruparon del modo esperado, por eso nuevos estudios deberán ser realizados, visando el refinamiento del instrumento. Las condiciones ambientales y personales fueron los estilos predominantes en esta muestra.

Palabras clave: Aprendizaje; estilos cognitivos; evaluación psicológica. 


\section{Introdução}

A aprendizagem pode ser concebida como uma ação contínua que abarca aspectos como ambiente, emoções, valores e constante aprimoramento. Pozo (1996) discute que o desafio da aprendizagem está na habilidade do sujeito representar mentalmente a realidade de modo a poder operar sobre ela. Nessa direção, pode-se dizer que a aprendizagem é um processo mental complexo que depende de uma diversidade de fatores para que se efetive.

Dentre as concepções acerca da aprendizagem, há aquelas que consideram que as estruturas neuronais trabaIham em conjunto de modo a favorecer ou potencializar o aprendizado. Essas estruturas são responsáveis por receber e analisar os estímulos externos, visando a interação com os conhecimentos prévios já armazenados na cognição. Desse modo, a busca por informações já conhecidas a fim de organizar novas informações faz da mente um processador (Woolfolk, 2000).

Autores como Sternberg (2000) consideram que a aprendizagem se concretiza quando há um processamento profundo de alguma nova informação. Nesse processo, as contingências ambientais são as promotoras da estimulação do raciocínio; assim sendo, quando uma nova informação é adquirida e armazenada na memória inicia-se o processamento mental. A memória pode ser compreendida como tendo diversas estruturas mentais ou processadores que seriam responsáveis pelos armazenamentos sensorial, da memória de trabalho e de longa duração.

Para Dembo (1988), Hannon e Daneman (2001) e Nori e Giusbert (2006), o armazenamento sensorial é responsável por preservar a informação até que esta seja transferida para a memória de trabalho. Na memória de trabalho a informação é codificada e armazenada por um curto período de tempo, até que seja enviada à memória de longa duração, que por sua vez, deve arquivar as novas informações de modo que todas as ocasiões em que o sujeito necessite acessar essas informações, elas possam estar disponíveis à recuperação.

No contexto formal de ensino é possível observar variados padrões de aprendizagem do aluno em atividades que envolvem leitura, compreensão de textos e tarefas. Esses padrões podem apresentar características que envolvem tanto o processamento profundo quanto o processamento mais superficial da informação (Gomes, 2011). Cabe destacar que além dos aspectos cognitivos envolvidos no processamento, há também as peculiaridades pessoais de cada indivíduo, sendo que estas também podem influenciar o modo como ocorre a aquisição do conhecimento.

Zhang e Sternberg (2005) ponderam que a maneira preferencial de processamento da informação e realização das tarefas, isto é, o perfil de processamento envolvido pode ser considerado estilo intelectual. Para os autores, um estilo intelectual é composto por fatores cognitivos (a tarefa de processar a informação está ligada a alguma função da cognição humana), afetivos, (o modo de se lidar com uma tarefa envolve sentimentos e sensações sobre a tarefa), fisiológicos (a informação sofre influência do modo como os nossos sentidos recepcionam e interpretam essa informação), psicológicos (dependente dos crivos psicológicos estabelecidos na relação com o meio) e sociológico (sofre influência da sociedade e consequentemente de sua forma de pensar).

Nessa mesma direção, é possível encontrar na literatura científica sobre os estilos de aprendizagem autores como Felder (2002), Felder e Spurlin (2005) e Litzinger, Lee, Wise e Felder (2007) que convergem para o entendimento que o modo preferencial de recepção e processamento da informação no contexto da aprendizagem pode ser denominado como estilo de aprendizagem. Concepção com certa semelhança também está presente nas publicações de Alonso, Gallego, e Honey (2002), Biggs, Kember, e Leung (2001), Cantwell e Moore (1998), Holland (1985) e Kolb (1984), dentre outros.

Contudo, embora exista o entendimento do estilo de aprendizagem como permanecendo ligado à natureza do processamento intelectual humano, ainda há incoerências no que tange à terminologia empregada. Tal fato é abordado por Zhang e Sternberg (2005) quando argumentam que a expressão estilos, empregada em diversas publicações, pode ser considerada geral. Não há uma única classificação, o que existe são variações na expressão, quais sejam, estilo de resolução problemas, estilo de processamento superficial e profundo, estilo de manejo de tempo, estilo de tomada de decisão para resolução de problemas, estilo da mente, estilo perceptual, estilo de pensamento, estilo intelectual, estilo cognitivo, estilo de aprendizagem, dentre tantos outros.

Zhang e Sternberg (2005) acrescentam que há uma diversidade de teorias e modelos sobre os estilos e dessa variação também se derivaram as expressões empregadas para os estilos. Os estilos por serem considerados multidimensionais, permitiram diferentes autores estabelecessem um perfil característico para cada estilo (fator/dimensão) encontrada em seu instrumento. Posto isto, a literatura científica sobre estilos é permeada por discrepâncias no modo como se compreende este construto.

Em síntese, é possível considerar que quando o comportamento envolve o processamento cognitivo e o perfil deste, estariam sendo trabalhados os estilos intelectuais ou estilos cognitivos, isto é, o perfil preferencial do processamento, considerados equivalentes a traços de comportamento/personalidade. Um traço de comportamento/personalidade caracterizaria o funcionamento do sujeito, como por exemplo pessoas com um comportamento mais autônomo teriam um estilo autônomo, uma pessoa com melhor processamento visual, teria um estilo visual predominando em suas ações, uma pessoa poderia ter um funcionamento mais pragmático, portanto, um estilo pragmático e assim por diante (ver Alonso \& cols, 2002; Zhang \& Sternberg, 2005).

Sob essa perspectiva, as investigações realizadas por Santos, Sisto e Martins (2003) indicaram que pode haver tendências diferenciadas no modo como as pessoas aprendem, conhecidas como estilos cognitivos (ou estilos intelectuais). Os estilos cognitivos refletem o automonitoramento 
do processo de aprender (funcionamento metacognitivo); por meio deles os indivíduos conseguem organizar e controlar tanto o processamento da informação como também as respostas emocionais. Assim sendo, os estilos cognitivos integrariam tanto os aspectos cognitivos quanto os afetivos envolvidos na aprendizagem. Os autores consideram, também, que os estilos cognitivos teriam o papel de mediadores entre a habilidade cognitiva e as características de comportamento/personalidade do sujeito. Desse modo, os estilos cognitivos discriminariam o limite entre os elementos da cognição e as características de comportamento/personalidade.

Acredita-se, contudo, que aspectos ou ações mais simples também estão relacionados à preferência do sujeito e, dessa forma, podem interferir no processo de aprendizagem. Dentre eles, pode-se citar o tempo dedicado ao estudo, o estudar com barulho ou silêncio, recursos empregados para aprender, condições pessoais do aprendiz, condições da socialização entre pares na realização das atividades, bem como características da tarefa a ser aprendida (Santos \& Mognon, 2010). De forma metafórica, imaginando-se uma fruta, poder-se-ia considerar os estilos intelectuais ou cognitivos como sendo a polpa e os estilos de aprendizagem como sendo a casca.

Sob essa perspectiva, pode-se dizer que não existe um quantum negativo ou positivo em relação aos estilos de aprendizagem (Santos \& Mognon, 2010). Constata-se, contudo, a prevalência de estilos que se diferenciam entre os escolares, isto quer dizer que um determinado perfil ou estilo para aprender não possui maior ou menor valor em relação ao outro. Com base nesse entendimento os educadores devem buscar a promoção do desenvolvimento dos diferentes estilos de aprendizagem nas situações de ensino em detrimento de privilegiarem determinados tipos de estilos. Assim, com o conhecimento sobre a diversidade acerca dos estilos de aprendizagem, os alunos poderiam monitorar melhor o próprio aprendizado, favorecendo uma otimização na aquisição do conhecimento.

Em âmbito nacional há que se citar os estudos de Gomes, Golino, Pinheiro, Miranda, e Soares (2011) e Gomes (2013) que buscaram validar a Escala de Abordagens de Aprendizagem (EABAP). As pesquisas foram realizadas com estudantes brasileiros do ensino fundamental e médio e, embora bem desenvolvidas, foram baseadas no modelo de processamento profundo e superficial, proposição teórica que difere da compreensão trazida na presente pesquisa.

Face ao apresentado, fica evidenciada a importância de se estudar os estilos de aprendizagem em âmbito nacional. No entanto, com o entendimento de que os estilos de aprendizagem diferem dos estilos intelectuais ou cognitivos por terem uma característica de menor profundidade (mas não de menor importância) no aprendizado, não foram recuperados, no contexto nacional, estudos sobre instrumentos de medida que se pautam nessa concepção. Há 16 anos atrás, Bariani, Sisto e Santos (2000) construíram uma escala para avaliar os estilos cognitivos, tendo por base o entendimento do estilo cognitivo como sendo algo mais relacionado ao traço de personalidade, concepção diferente, portanto, da apresentada no presente trabalho para o entendimento dos estilos de aprendizagem.

Na literatura internacional há diversas de publicações que trazem diferentes formas de avaliação desse construto. Dentre os instrumentos pesquisados, apresentados em ordenação cronológica, estão o Learning Style Inventory - LSI de Kolb's (1976), o Self-Directed Search - SDS de Holland (1985), o Kirton Adaption-Innovation Inventory - KAll de Kirton (1987), o Style of Learning and Thinking - SOLAT de Torrance, McCarthy e Kolesinski (1988), o The Myers-Briggs Type Indicator - MBTI de Myers e McCaulley (1988), o Study Process Questionnaire - SPQ (Biggs's, 1987; 1992), o Studying Inventory de Wilson, Smart e Watson (1996), o Strategic Flexibility Questionnaire de Cantwell e Moore (1998), o Study Process Questionnaire: R-SPQ-2F de Biggs, Kember e Leung (2001), dentre outros.

Tendo em vista a carência de instrumentos e de pesquisas brasileiras que focaram os estilos de aprendizagem e diante das considerações apresentadas, o presente estudo visou identificar evidências de validade relativa à análise da estrutura interna dos itens de uma escala de estilos de aprendizagem direcionada a estudantes do ensino fundamental. Também foram identificados os estilos de aprendizagem predominantes nos estudantes participantes.

\section{Método}

\section{Participantes}

Os participantes do presente estudo foram alunos do ensino fundamental, do $3^{\circ}$ ano ao $9^{\circ}$ ano, totalizando 565 estudantes provenientes de escolas públicas estaduais e municipais de três cidades do estado do Paraná. O gênero feminino representou $47,8 \%(\underline{n}=270)$ e o masculino $52,3 \%$ ( $\underline{n}=295)$. A idade média dos estudantes foi de 10 anos e 2 meses $(\underline{D P}=1,68)$, sendo a idade mínima 7 anos e máxima de 17 anos.

\section{Instrumentos}

O instrumento utilizado para a coleta deste estudo foi a Escala de Avaliação de Estilos de Aprendizagem, elaborada por Santos no ano de 2006, destinada à descrição dos estilos preferenciais. A escala avalia cinco dimensões dos estilos de aprendizagem, condições ambientais, condições sociais, condições instrumentais, condições pessoais e condições da atividade. A Escala é composta por 80 questões, com quatro alternativas, em escala likert, sempre (4 pontos), muitas vezes ( 3 pontos), poucas vezes (2 ponto) e nunca (1 ponto).

A dimensão 1, condições ambientais, diz respeito aos estímulos físicos do ambiente imediato, tais como, iluminação, ventilação, ruído, tempo, espaço (tipo de ambiente formal ou informal e sua estrutura física) e ficou composta 
por 22 itens $(2,6,10,11,28,30,39,41,42,45,48,49,56$, $58,59,62,67,68,69,70,71$ e 75). Para exemplificar esta dimensão podem-se citar os itens 2 e 6: "Prefiro estudar em silêncio" e "Rádio ligado atrapalha minha aprendizagem", respectivamente. A dimensão 2, condições sociais, diz respeito à maneira preferencial de realização da tarefa, considerando-se os envolvidos no processo de ensino-aprendizagem (aluno-colegas-professor) e ficou composta por 14 itens (9, $12,15,21,27,31,34,36,51,54,57,61,77$ e 80). Os itens 9 e 12 podem exemplificar esta dimensão: "Prefiro realizar tarefas em grupo" e "Gosto de trocar ideias com meus colegas sobre o assunto que estamos estudando", respectivamente. A dimensão 3, condições instrumentais, diz respeito à preferência por recursos didáticos e tecnológicos durante a situação de aprendizagem (recursos como jogos, revistas, jornais, livros, apostilas, lousa, retroprojetor, computador, TV e vídeo) e ficou composta por 12 itens $(14,16,23,32,35$, $37,40,52,55,60,72$ e 73). Exemplos que avaliam esta dimensão podem ser ilustrados pelos itens 14 e 16: "Aprendo pouco por meio de jogos" e "O uso de retroprojetor facilita a compreensão da matéria”, respectivamente. A dimensão 4, condições pessoais, diz respeito às preferências sensoriais, perceptuais e cognitivas do aprendiz na execução de uma tarefa (o fazer, observar, ouvir, ler, o ritmo de execução e resolução do problema) e ficou composta por 20 itens (1, 4, $7,13,18,20,22,24,26,38,43,44,46,50,53,63,64,66$, 76 e 79). Os itens 1 e 4 podem avaliar, respectivamente, esta dimensão: "Aprendo melhor anotando durante as aulas" e "Preparar trabalhos para apresentar em classe atrapalha minha aprendizagem". Por fim, a dimensão 5, condições da atividade, diz respeito à maneira preferencial de formato e proposição da atividade (atividades mais detalhadas, dirigidas, organizadas, claras, fechadas, livres, convencionais, controladas, originais e/ou criativas) e ficou composta por 12 itens $(3,5,8,17,19,25,29,33,47,65,74$ e 78). Exemplos como os itens 3 e 5: "Aprendo melhor com as explicações do professor" e "Aprendo melhor quando a matéria está bem detalhada" avaliam, respectivamente, esta dimensão.

Vale destacar que os itens em suas várias dimensões foram propostos com base na descrição apresentada na literatura sobre o tema. Sua alocação nas dimensões foi julgada por especialistas (alunos de pós-graduação stricto-sensu da área de avaliação psicológica). O critério para aceitação do item foi a concordância de $80 \%$ entre os juízes.

\section{Procedimentos de Coleta de Dados}

Os responsáveis legais pelos participantes assinaram um termo de consentimento livre e esclarecido, consentindo na participação do menor. A coleta aconteceu de forma coletiva em sala de aula, em dia e horário agendado pelas instituições coparticipantes. A coleta de dados foi realizada e acompanhada sempre por um dos pesquisadores, com uma duração aproximada de 30 minutos, entre a explicação dos questionários e tempo para resposta dos mesmos.
Cabe mencionar que foi realizada uma aplicação piloto com aproximadamente 100 alunos, sendo estes dos $1^{\circ}$ e $2^{\circ}$ anos. Contudo, estes demonstraram falta de conhecimento acerca de várias palavras do instrumento (ex. Item 13 - Ter liberdade para realizar minhas tarefas facilita minha aprendizagem; item 74 - Tarefas detalhadas facilitam minha aprendizagem, dentre outros). No caso dos alunos do $1^{\circ}$ ano, estes não estavam alfabetizados e mesmo a aplicação ocorrendo de forma individual e o aplicador procedendo à leitura da questão, havia pouco entendimento acerca do item por parte da criança. No caso dos alunos do $2^{\circ}$ ano, apesar de já estarem alfabetizados, ainda apresentaram grande dificuldade para o entendimento dos itens. Posto isto, optou-se por trabalhar apenas com os alunos do $3^{\circ}$ ao $9^{\circ}$ ano.

\section{Análise de Dados}

Para uma melhor operacionalização dos objetivos os dados foram organizados em planilha Excel e submetidos à análise das estatísticas inferencial (Análise fatorial exploratória) e descritiva (médias e Desvio-padrão), com o intuito de atender aos objetivos propostos pela pesquisa.

\section{Resultados}

A fim de verificar a possibilidade de aplicação da análise fatorial exploratória, foi efetuado o Teste de Esfericidade de Bartlett, o qual indicou correlação entre os itens $(\underline{x} \underline{2}[3160$; $\underline{N}=565]=9050,213 ; \underline{p}<0,001)$ e, portanto, adequabilidade ao uso da análise fatorial. A adequação da amostra pode ser aferida pelo índice de Kaiser-Meyer-Olkin (KMO) que foi de 0,858 . Após a análise fatorial, por componentes principais e rotação varimax, configurou-se uma estrutura de quatro dimensões para a escala, com eigenvalues acima de 1,0, capaz de explicar $37,29 \%$ da variância total. As dimensões se agruparam da seguinte forma, Dimensão 1 - Condições ambientais e Condições pessoais, com 22 itens (1, 2, 6, 7, $11,18,20,22,24,30,46,49,50,53,56,58,59,62,63,64$, 66 e 67); Dimensão 2 - Condições sociais, com 11 itens (9, 12, 15, 27, 31, 34, 51, 54, 57, 61 e 77); Dimensão 3 - Condições instrumentais, com quatro itens (23, 35, 40 e 73), e Dimensão 4 - Condições de atividade, com dois itens (65 e 78). A Tabela 1 mostra a distribuição dos itens por dimensão e suas respectivas cargas fatoriais.

Para a análise fatorial exploratória, a menor carga fatorial foi de 0,367 e a maior carga fatorial foi de 0,714. Para a Dimensão 1 - condições ambientais e pessoais o alfa de Cronbach encontrado foi de 0,88 , para a Dimensão 2 - condições sociais 0,84 , para a Dimensão 3 - condições instrumentais o valor encontrado foi 0,64 e para a Dimensão 4 - condições de atividade o valor encontrado foi de 0,28.

Após a análise, a escala de 80 itens foi reduzida para 39 itens, os itens 3, 19, 25 e 29 (condições de atividade), itens 32 e 52 (condições instrumentais) foram excluídos por carregarem para a Dimensão 1. O item 21 (condições so- 
Tabela 1. Distribuição dos itens por Dimensão e suas respectivas cargas fatoriais.

\begin{tabular}{|c|c|c|c|c|c|c|}
\hline Itens & & 1 & 2 & 3 & 4 & Comunalidade \\
\hline 1 & Aprendo melhor anotando durante as aulas. & 0,395 & & & & 0,372 \\
\hline 2 & Prefiro estudar em silêncio. & 0,593 & & & & 0,426 \\
\hline 6 & Rádio ligado atrapalha minha aprendizagem. & 0,411 & & & & 0,255 \\
\hline 7 & Aprendo melhor quando participo das aulas. & 0,514 & & & & 0,363 \\
\hline 9 & Prefiro realizar tarefas em grupo. & & 0,650 & & & 0,457 \\
\hline 11 & Prefiro estudar em lugares silenciosos & 0,703 & & & & 0,561 \\
\hline 12 & $\begin{array}{l}\text { Gosto de trocar ideias com meus colegas sobre o assunto que } \\
\text { estamos estudando. }\end{array}$ & & 0,476 & & & 0,293 \\
\hline 15 & Aprendo mais quando sou ajudado por meus colegas. & & 0,650 & & & 0,491 \\
\hline 18 & Prefiro atividades em que eu possa criar coisas novas. & 0,500 & & & & 0,393 \\
\hline 20 & Sou organizado quando estudo. & 0,645 & & & & 0,461 \\
\hline 22 & Tenho mais facilidade para aprender ouvindo. & 0,402 & & & & 0,318 \\
\hline 23 & Assistir a vídeos sobre a matéria atrapalha minha compreensão. & & & 0,513 & & 0,294 \\
\hline 24 & Gosto de atividades que me façam pensar. & 0,615 & & & & 0,402 \\
\hline 27 & Prefiro aprender interagindo com meus colegas de classe. & & 0,629 & & & 0,564 \\
\hline 30 & Prefiro estudar numa biblioteca. & 0,367 & & & & 0,228 \\
\hline 31 & Aprendo melhor em situações de grupo. & & 0,633 & & & 0,561 \\
\hline 34 & Aprendo mais quando ajudo meus colegas. & & 0,516 & & & 0,379 \\
\hline 35 & Ter que usar o computador dificulta minha aprendizagem. & & & 0,441 & & 0,291 \\
\hline 40 & Consultar dicionário prejudica minha aprendizagem. & & & 0,558 & & 0,379 \\
\hline 46 & Gosto de realizar atividades que dependem da minha imaginação. & 0,388 & & & & 0,397 \\
\hline 49 & Prefiro estudar em ambientes bem iluminados. & 0,506 & & & & 0,307 \\
\hline 50 & Costumo ter ideias novas enquanto aprendo. & 0,523 & & & & 0,474 \\
\hline 51 & $\begin{array}{l}\text { Fico contente quando aquilo que penso é parecido com o que meus } \\
\text { colegas pensam. }\end{array}$ & & 0,491 & & & 0,363 \\
\hline 53 & Costumo ter ideias novas. & 0,576 & & & & 0,372 \\
\hline 54 & Estudar em grupo me faz aprender melhor. & & 0,714 & & & 0,638 \\
\hline 56 & Prefiro estudar sentado. & 0,586 & & & & 0,390 \\
\hline 57 & Tenho mais facilidade de aprender com meus colegas. & & 0,692 & & & 0,543 \\
\hline 58 & Qualquer barulho prejudica minha aprendizagem. & 0,442 & & & & 0,401 \\
\hline 59 & Prefiro estudar em ambientes calmos. & 0,673 & & & & 0,481 \\
\hline 61 & Situações de competição facilitam minha aprendizagem. & & 0,432 & & & 0,376 \\
\hline 62 & TV ligada atrapalha minha aprendizagem. & 0,418 & & & & 0,452 \\
\hline 63 & Aprendo mais quando tenho que falar sobre o assunto. & 0,414 & & & & 0,357 \\
\hline 64 & Aprendo melhor fazendo. & 0,619 & & & & 0,452 \\
\hline 65 & Exercícios práticos auxiliam minha aprendizagem. & & & & 0,408 & 0,352 \\
\hline 66 & Gosto de aprender ouvindo as explicações. & 0,491 & & & & 0,308 \\
\hline 67 & Aprendo melhor em dias quentes. & 0,366 & & & & 0,296 \\
\hline 73 & Sinto dificuldade para estudar em livro. & & & 0,608 & & 0,450 \\
\hline 77 & $\begin{array}{l}\text { Concordo com as ideias dos meus colegas sobre os temas que } \\
\text { estudamos. }\end{array}$ & & 0,487 & & & 0,398 \\
\hline 78 & Ter que seguir instruções atrapalha minha aprendizagem. & & & & 0,396 & 0,477 \\
\hline
\end{tabular}

ciais) foi excluído por carregar em mais de uma dimensão, os itens 37 e 55 (condições instrumentais) e o item 33 (condições de atividade) foram excluídos por não carregarem na Dimensão 2. Os itens 4, 43 e 76 (condições pessoais), itens 36 e 80 (condições sociais), itens 48, 68, 69 e 70 (condições ambientais) e o item 78 (condições de atividade) foram eliminados por não carregarem na Dimensão 3. Os itens 10 , 28, 41, 62 e 71 (condições ambientais), itens 13, 22, 38, 44 (condições pessoais), itens 60 e 72 (condições instrumentais) e itens 65 e 78 (condições de atividade) excluídos por não carregarem na Dimensão 4. Os itens restantes, 5, 8, 14, $16,17,39,45,47,74,75$ e 79 também foram excluídos, entretanto, por não apresentarem carga fatorial para as quatro dimensões forçadas. A Tabela 2 apresenta os 39 itens agrupados de acordo com a congruência em seus fatores e seus respectivos alfas de $\underline{\text { Cronbach. }}$. 
Tabela 2. Distribuição dos itens, suas respectivas dimensões teóricas e alfa de Cronbach

\begin{tabular}{|c|c|c|c|}
\hline Itens & & Dimensão & Alfa de Cronbach \\
\hline 1 & Aprendo melhor anotando durante as aulas. & \multirow{22}{*}{$\begin{array}{c}\text { Condições Pessoais e } \\
\text { Ambientais }\end{array}$} & \multirow{22}{*}{0,88} \\
\hline 2 & Prefiro estudar em silêncio. & & \\
\hline 6 & Rádio ligado atrapalha minha aprendizagem. & & \\
\hline 7 & Aprendo melhor quando participo das aulas. & & \\
\hline 11 & Prefiro estudar em lugares silenciosos & & \\
\hline 18 & Prefiro atividades em que eu possa criar coisas novas. & & \\
\hline 20 & Sou organizado quando estudo. & & \\
\hline 22 & Tenho mais facilidade para aprender ouvindo. & & \\
\hline 24 & Gosto de atividades que me façam pensar. & & \\
\hline 30 & Prefiro estudar numa biblioteca. & & \\
\hline 46 & Gosto de realizar atividades que dependem da minha imaginação. & & \\
\hline 49 & Prefiro estudar em ambientes bem iluminados. & & \\
\hline 50 & Costumo ter ideias novas enquanto aprendo. & & \\
\hline 53 & Costumo ter ideias novas. & & \\
\hline 56 & Prefiro estudar sentado. & & \\
\hline 58 & Qualquer barulho prejudica minha aprendizagem. & & \\
\hline 59 & Prefiro estudar em ambientes calmos. & & \\
\hline 62 & TV ligada atrapalha minha aprendizagem. & & \\
\hline 63 & Aprendo mais quando tenho que falar sobre o assunto. & & \\
\hline 64 & Aprendo melhor fazendo. & & \\
\hline 66 & Gosto de aprender ouvindo as explicações. & & \\
\hline 67 & Aprendo melhor em dias quentes. & & \\
\hline
\end{tabular}

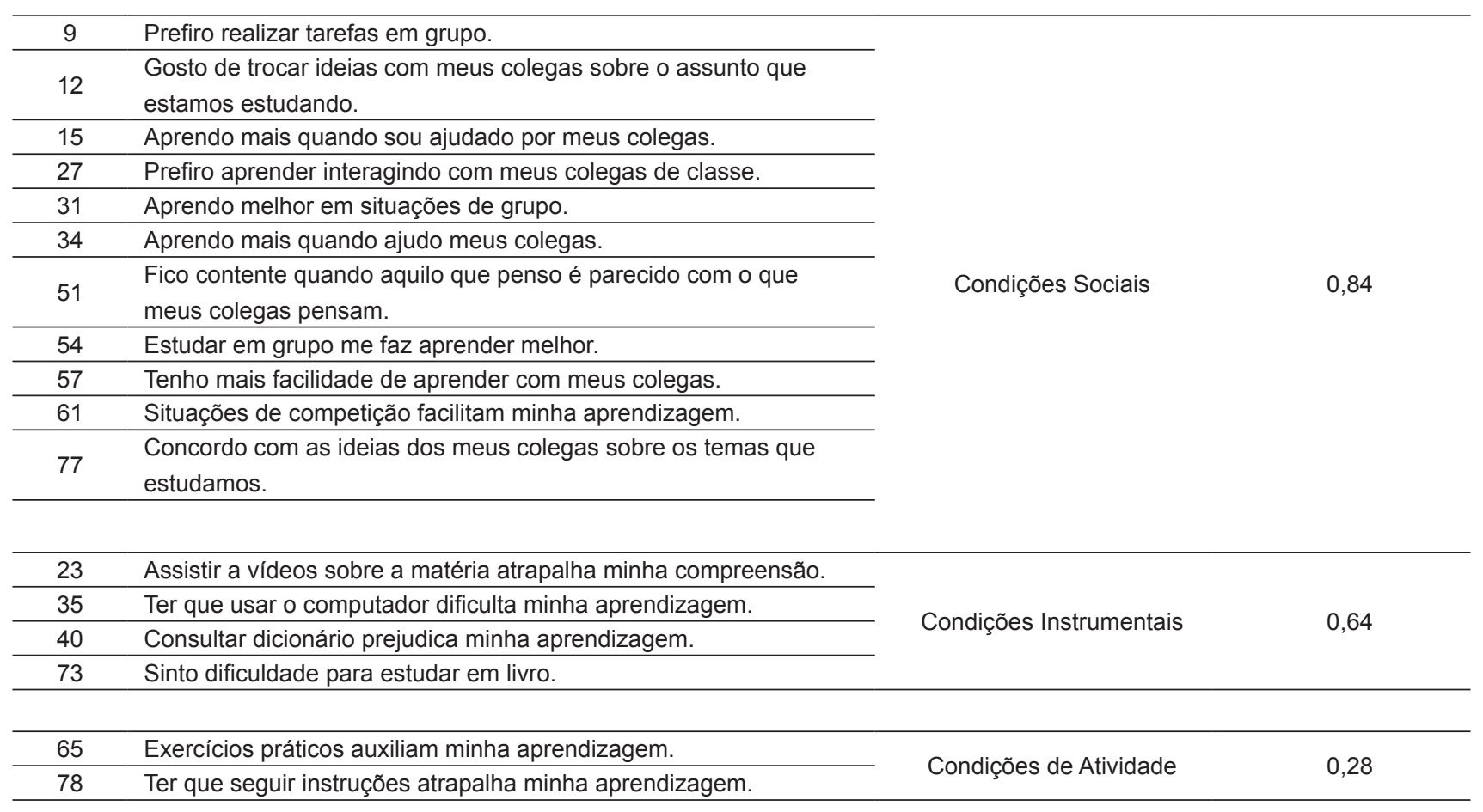

Para atender ao segundo objetivo foram identificados os estilos de aprendizagem utilizados pelos estudantes do ensino fundamental e também calculada a estatística descritiva para as subescalas, condições ambientais e pessoais, sociais, instrumentais e de atividades, descritas na Tabela 3.

De acordo com a Tabela 3, a média de pontos obtidos para a Dimensão condições ambientais e pessoais foi de 59,83 pontos ( $\underline{D P}=12,85)$, com pontuação mínima de 22 $(\underline{n}=1,0,3 \%)$ e máxima de $88(\underline{n}=1,0,2 \%)$ maior pontuação possível na escala. A partir desta análise, é presumível observar que $36,1 \%$ dos estudantes $(\underline{n}=204,0,2 \%)$ apresentaram pontuação superior à média de pontos alcançados, demonstrando importância quanto às condições ambientais e pessoais. 
Tabela 3. Distribuição das médias, desvio padrão, pontuação mínima e máxima das subescalas para os estilos de aprendizagem.

\begin{tabular}{cccccc}
\hline Subescala & $\begin{array}{c}\text { Variação da } \\
\text { Pontuação }\end{array}$ & $\begin{array}{c}\text { M de pontos na } \\
\text { Escala }\end{array}$ & DP & $\begin{array}{c}\text { Pontuação } \\
\text { Mínima }\end{array}$ & $\begin{array}{c}\text { Pontuação } \\
\text { Máxima }\end{array}$ \\
\hline Condições Ambientais e Pessoais & $0-88$ & 59,83 & 12,85 & 22 & 78 \\
\hline Condições Sociais & $0-44$ & 28,48 & 7,64 & 74 & 0 \\
\hline Condições Instrumentais & $0-16$ & 5,57 & 2,98 & 0 & 8 \\
\hline Condições de Atividade & $0-8$ & 4,42 & 1,67 & 8 \\
\hline
\end{tabular}

A análise das condições sociais indicou uma média de pontos de 28,48 ( $\underline{D P}=7,64)$, com pontuação mínima de $7(\underline{n}=1,0,2 \%)$ e máxima de $44(\underline{n}=3,0,5 \%)$. É possível destacar que $45,6 \%$ dos estudantes $(\underline{n}=258)$ atingiram uma pontuação superior à média, dando importância às condições sociais. Para as condições instrumentais, a pontuação média atingida foi de $5,57(\underline{\mathrm{DP}}=2,98)$, com a pontuação mínima de zero ( $\underline{n}=38,6,7 \%)$ e máxima de $16(\underline{n}=1,0,2 \%)$, maior pontuação possível na escala. Destaca-se que $46 \%$ dos estudantes $(\underline{n}=260)$ pontuaram acima da média quanto às condições instrumentais. Quanto às condições de atividade, a pontuação média apresentada foi de 4,42 ( $\underline{\mathrm{DP}}=1,67)$, com pontuação mínima de zero ( $\underline{n}=5,0,9 \%)$ e com pontuação máxima de 8 ( $\underline{n}=27,4,8 \%)$, demostrando desta forma que $46 \%$ dos alunos ( $\underline{n}=260)$ atingiram escore acima da média quanto às condições de atividade.

\section{Discussão}

O contexto escolar é permeado por uma diversidade de interesses e experiências de aprendizagem por parte do alunado. Contudo, embora seja um ambiente profícuo ao despertar do conhecimento, Burton, Taylor, Dowling e Lawrence (2009) argumentam que as salas de aula podem refletir um abandono escolar produto de uma educação deficitária desde as séries iniciais, o que reflete na qualidade da formação no ensino superior. Os autores chamam a atenção para uma geração de alunos denominados por eles de 'geração net' que apesar de terem uma facilidade de acesso à informação, poucos de fato são estratégicos no momento do estudo. Com esse ponto de partida na discussão, argumenta-se que compreender os estilos de aprendizagem e o perfil de estilos de aprendizagem do alunado poderia ser de suma importância para fomentar a implementação, por exemplo, de um comportamento mais estratégico no momento da aprendizagem.

Estudiosos do tema consideram que as pessoas concebem e lidam com a aprendizagem de forma diferenciada e personalizada (Dias, Sauaia, \& Yoshizaki, 2013, Moura Filho, 2013; Oliveira, Negreiros, \& Neves, 2015; Valladas, 2007; Zhang \& Sternberg, 2005). Assim sendo, cada um utiliza suas habilidades de forma específica. Cada pessoa é submetida a contextos contingenciais que fazem com que cada uma tenha uma construção tanto como sujeito único quanto como sujeito grupal. Dessa maneira, parece plausível que cada um tenha um estilo particular para a aquisição, armazenamento e posterior recuperação da informação. Mais precisamente, em relação ao ambiente educacional, é possível dizer que cada aluno tem uma forma preferencial de estudar, ou seja, tem um estilo de aprendizagem predominante.

Embora estudar os estilos de aprendizagem seja relevante, não é algo simples. Os estilos de aprendizagem, com suas diversas bases teóricas, apresentam muitas discrepâncias em seus postulados, havendo divergências, inclusive, quanto à sua classificação. No entanto, há um aspecto comum quanto à compreensão de que os estilos de aprendizagem são atinentes ao processo cognitivo (Zhang \& Sternberg, 2005).

O modo preferencial do aluno para adquirir e processar o conhecimento seria a definição de estilos de aprendizagem. Como já mencionado, na literatura científica os estilos de aprendizagem são tidos como perfil de personalidade ou como perfil comportamental de funcionamento. No entanto, no presente trabalho assumiu-se a posição de que os estilos de aprendizagem se referem a comportamentos expressos de preferência nas situações de aprendizagem. Por exemplo, algumas pessoas conseguem estudar em ambientes mais agitados e ruidosos, enquanto outras somente conseguem concretizar o aprendizado em ambientes mais tranquilos. Em outro sentido, é sabido que alguns aprendem melhor quando as atividades propostas são predominantemente grupais ou individuais.

Nessa perspectiva, o foco não seria no perfil cognitivo ou estilo cognitivo/intelectual, ou mesmo no perfil de processamento (ver Sisto \& cols., 2000; Valadas, 2007; Zhang \& Sternberg, 2005, dentre outros), o que se estaria focando é simplesmente o estilo de aprendizagem (preferência pelos meios dos quais haverá a aquisição do conhecimento). Com a identificação do perfil da amostra foi possível constatar que o perfil predominante foi aquele que agregava tanto as condições ambientais quanto as condições pessoais. Nesse perfil, há alunos que, no momento do estudo, valorizam tanto os estímulos físicos do ambiente imediato (iluminação, ventilação, ruído, tempo, espaço, tipo de ambientes e estrutura física) quanto aqueles que salientam as preferências sensoriais, perceptuais e cognitivas do aprendiz na execução de uma tarefa (o fazer, observar, ouvir, ler, o ritmo de execução e resolução do problema). 
Esse dado não causa estranheza, pois para alunos do ensino fundamental, em especial os da primeira etapa, o ambiente físico pode ser mais importante para que o seu aprendizado se concretize. Assim, ambiente ruidoso, mal iluminado e/ou desorganizado, pode dificultar a atenção e a concentração de alunos menores, que ainda estão aprendendo a regular o próprio comportamento no momento do estudo, não conseguindo filtrar o barulho para conseguir se concentrar.

Também parece salutar que nessa etapa de escolarização a condição pessoal do sujeito também assuma importância, pois nessa fase escolar os alunos fazem muito uso da observação, são dependentes do ritmo de execução da tarefa, também aprendem melhor quando concretizam (fazem), por assim dizer, a tarefa. Ao se considerar a média de pontos na escala e o número de itens, percebe-se que a condição instrumental foi a menos prevalente entre os alunos da amostra. Os alunos participantes não expressaram preferência por recursos didáticos e tecnológicos durante a situação de aprendizagem. Hipotetiza-se, contudo, a possibilidade de que o aluno, à medida que vai ficando mais velho, se interesse mais por recursos tecnológicos, valorizando mais os recursos didáticos, tornando-se mais orientado à condição ambiental. Posto isto, seria necessário a realização de investigações que pudessem elucidar se, de fato, essa hipótese se confirmaria.

No que tange à análise da estrutura fatorial exploratória da escala, os dados permitiram identificar que novos estudos serão necessários para identificar melhor a organização das dimensões, quais sejam, condições pessoais e ambientais, condição social, condição instrumental e condição de atividade. Esta última, em especial, necessitará passar por uma nova reformulação. A escala como um todo terá que ser novamente aplicada em novas amostras para melhor compreender os estilos de aprendizagem de alunos do ensino fundamental. Embora tenha se excluído a participação dos alunos dos anos iniciais, conforme relatado anteriormente, considera-se que os itens ainda podem ficam mais claros, de forma a serem mais acessíveis para a compreensão dos alunos. Com isso, quem sabe, poder-se-ia estender o uso aos alunos dos primeiros anos do ensino fundamental.

Cabe esclarecer que não se calculou um valor de alfa da escala como um todo, haja vista que cada item da escala representa um estilo de aprendizagem, e um estilo não é melhor ou pior do que o outro. Dos 80 itens estudados, apenas 39 foram aproveitados, sendo que dois deles compõem a subescala de condições de atividade, aquela que não atingiu valor aceitável do alfa.

Ainda que os valores do alfa das subescalas referentes às condições pessoais e ambientais, à condição social e à condição instrumental tenham sido aceitáveis, compreende-se que ainda há a necessidade de revisão do instrumento. Em razão disso, é importante mencionar que novas investigações estão sendo realizadas para que este instrumento possa ser empregado futuramente, de forma confiável, para a avaliação dos estilos de aprendizagem de alunos do ensino fundamental. Considera-se, portanto, que a versão obtida no presente estudo ainda é preliminar, mas representa um avanço na direção pretendida de construir uma escala que indique as preferências na forma de aprender dos estudantes.

\section{Referências}

Alonso, C.M., Gallego, D.J. \& Honey, P. (2002). Los estilos de aprendizaje: procedimientos de diagnóstico y mejora. Madrid: Mensajero.

Bariani, I.C.D., Sisto, F.F., \& Santos, A.A.A. (2000). Construção de um instrumento de avaliação de estilos cognitivos. Em F.F. Sisto, E.T.B. Sbardelini \& R. Primi (Orgs.), Contextos e questões da avaliação psicológica (173-188). São Paulo: Casa do Psicólogo.

Biggs, J.B. (1987). Student Approaches to Learning and Studying Australian Council for Educational Research, Hawthorn.

Biggs, J.B. (1992). Why and How Do Hong Kong Students Learn? Using the Learning and Study Process Questionnaires. Education Paper, (14), Faculty of Education, The University of Hong Kong.

Biggs, J.B., Kember, D., \& Leung, D. (2001). The revised two-factor Study Process Questionnaire: R-SPQ-2F. British Journal of Educational Psychology, 71(1), 133- 149.

Burton, L.J, Taylor, J.A., Dowling, D.G., \& Lawrence, J. (2009). Learning approaches, personality and concepts of knowledge of first-year students: mature-age versus school leaver. Studies in Learning, Evaluation, Innovation and Development, 6(1), 65-81.

Cantwell, R.H. \& Moore, P.J. (1998). Relationships among control beliefs, approaches to learning, and the academic performance of final-year nurses. Journal of Educational Research, 44(1), 98-102.

Dembo, M. H. (1988). Applying educational psychology in the classroom (3th ed.) New York: Logman Publishing Group.

Dias, G.P.P., Sauaia, A.C.A., \& Yoshizaki, H.T.Y. (2013). Estilos de aprendizagem Felder-Silverman e o aprendizado com jogos de empresa. Revista de Administração de Empresas, 53(5), 469-484.

Felder, R. (2002). Index of Learning Styles Page. Acesso em 31 de julho de 2013. Disponível em: http://www2.ncsu.edu/unity/lockers/ users/f/felder/public/RMF. html

Felder, R.M. \& Spurlin, J.E. (2005). Applications, reliability, and validity of the index of learning styles. International Journal of Engineering Education, 21(1), 103-112.

Gomes, C.M.A. (2011). Abordagem profunda e abordagem superficial à aprendizagem: diferentes perspectivas do rendimento escolar. Psicologia: Reflexão e Crítica, 24(3), 479-488. 
Gomes, C.M.A. (2013). A Construção de uma medida em abordagens de aprendizagem. Psico - RS, 44 (2), 193-203.

Gomes, C.M.A., Golino, H.F., Pinheiro, C.A.R., Miranda, G.R. \& Soares, J. (2011). Validação da escala de abordagens de aprendizagem (EABAP) em uma amostra brasileira. Psicologia: Reflexão e Crítica, 24(1), 19-27.

Hannon, B. \& Daneman, M. (2001). A new tool for measuring and understanding individual differences in the component processes of reading comprehension. Journal of Educational Psychology, 93(1), 103-128.

Holland, J.L. (1985). Making vocational choices: A theory of vocational personalities and work environments (2nd ed.), Prentice-Hall, Englewood Cliffs, NJ.

Kirton, M.J. (1987). Cognitive styles and creativity. Em S. G. Isaksen (Org.), Frontiers in creativity research: Beyond the basics (pp. 282304). Buffalo, NY: Bearly Limited.

Kolb, D.A. (1976). The learning style inventory: Technical manual. McBer: Boston, MA.

Kolb, D.A. (1984). Experiential learning: Experience as the source of learning and development. Englewood Cliffs: Prentice Hall.

Litzinger, T.A., Lee, S.H., Wise, J.C. \& Felder, R.M. (2007). A Psychometric study of the index of learning styles. Journal Engineering Education, 96(4), 309-319.

Moura Filho, A.C.L. (2013). Pessoal e intransferível: a relevância dos estilos de aprendizagem nas aulas de línguas estrangeiras. Revista Brasileira de Linguística Aplicada, 13(1), 313-343.

Myers, I. B. \& McCaulley, M. H. (1988). Manual: a guide to the development and use of the myers-briggs type indicator. Consulting Psychologists. Palo Alto: CA.

Nori, R. \& Giusberti, F. (2006). Predicting cognitive styles from spatial abilities. American Journal of Psychology, 119(1), 67-86.
Oliveira, M.F.A., Negreiros, J. G. M., \& Neves, A. C. (2015). Condicionantes da aprendizagem da matemática: uma revisão sistêmica da literatura. Educação e Pesquisa, Ahead of print. Disponível em: http://www.scielo.br/pdf/ep/2015nahead/15179702-ep-s1517-97022015051533.pdf

Pozo, J.I. (1996). Estratégias de aprendizagem. Em C. Coll, J. Palácios \& A. Marchesi (Orgs.), Desenvolvimento psicológico e educação: Psicologia da educação (pp. 176-197). Porto Alegre: Artes Médicas.

Santos, A.A.A. \& Mognon, J.F. (2010). Estilos de aprendizagem em estudantes universitários. Boletim de Psicologia, 60(133), 229-241.

Santos, A.A.A., Sisto, F.F., \& Martins, R.M.M. (2003). Estilos cognitivos e personalidade: um estudo exploratório de evidências de validade. Psico-USF, 8(1), 11-19.

Sternberg, R.J. (2000). Psicologia cognitiva. Tradução Maria Regina Borges Osório. Porto Alegre: Artes Médicas.

Torrance, E.P., McCarthy, B., \& Kolesinski, M.T. (1988). Style of learning and thinking. Scholastic Testing Service: Bensenville, IL.

Valadas, S.C.A.T.S. (2007). Sucesso acadêmico e desenvolvimento cognitivo em estudantes universitários: estudo das abordagens e concepções de aprendizagem. Tese de doutorado. Universidade de Algarve. Portugal.

Wilson, K.L., Smart, R.M., \& Watson, R. J. (1996). Gender differences in approaches to learning in first year psychology students. British Journal of Educational Psychology, 66(1), 59-71.

Woolfolk, A. (2000). Psicologia da educação. Porto Alegre: Artmed.

Zhang, L. \& Sternberg, R.J. (2005). A threefold model of intellectual styles. Educational Psychology Review, 17(1), 1-53.
Recebido em: 09/12/2014

Reformulado em: 28/01/2016

Aprovado em: 05/02/2016 


\section{Sobre os autores}

Katya Luciane de Oliveira (katyauel@gmail.com)

Psicóloga. Mestre em Psicologia pelo Programa de Estudos de Pós-graduação Stricto-Sensu em Psicologia da Universidade São Francisco. Doutora em Psicologia, Desenvolvimento Humano e Educação pela Faculdade de Educação da Unicamp. Professora adjunta do curso de Psicologia e do Programa de Mestrado em Educação da Universidade Estadual de Londrina.

Acácia Aparecida Angeli dos Santos (acacia.angeli@gmail.com)

Psicóloga. Doutora em Psicologia Escolar e do Desenvolvimento Humano pela USP, docente da graduação no curso de psicologia e no Programa de Pós-graduação Stricto-sensu em Psicologia, da Universidade São Francisco-SP. Bolsista produtividade do CNPq.

Fabio Alexandre Pereira Scacchetti (fabioscacchetti@utfpr.edu.br)

Engenheiro Têxtil. Especialista em Gestão Empresarial pela Universidade do Vale dos Sinos. Mestre em Educação pela Universidade Estadual de Londrina. Doutorando da Universidade do Minho. Docente do curso de Engenharia Têxtil na Universidade Tecnológica Federal do Paraná. 